\title{
A Influência dos preços sobre a demanda domiciliar por frutas e hortaliças: uma análise por classes de renda
}

\author{
Maria Micheliana da Costa Silva ${ }^{1}$
}

\begin{abstract}
Alexandre Bragança Coelho²
Resumo: Este estudo buscou analisar a demanda por frutas e hortaliças nos domicílios brasileiras usando os microdados da Pesquisa de Orçamentos Familiares (POF/ IBGE) de 2008/2009 para diferentes níveis de renda per capita. Especificamente, procurou-se verificar se reduções no custo de aquisição desses alimentos são eficazes para que os brasileiros consumam as quantidades recomendadas. Para tanto, estimou-se um sistema de demanda para 25 itens dessa categoria, por meio do modelo QUAIDS com a correção dos gastos nulos pelo procedimento de Shonkwiller e Yen. Verificou-se que para incentivar o consumo de frutas e hortaliças, reduções de preço são mais eficazes do que melhorias de renda das famílias. Entretanto, os resultados mostram que para que os domicílios mais pobres consigam elevar a aquisição diária por adulto equivalente ao nível de consumo adequado, a redução de preços deveria ser bem elevada, de cerca de $55 \%$.
\end{abstract}

Palavras-chave: Demanda. Modelo QUAIDS. Frutas e hortaliças.

\begin{abstract}
This paper analyzed the fruits and vegetables demand using microdata from the 2008/2009 Household Budget Survey (POF/IBGE), for different income classes. We aimed to assess whether price reductions are effective in promoting the recommended daily intake. Thus, we estimated a demand system for 25 goods, using the QUAIS model and the Shonkwiller e Yen procedure. We have found that to promote the fruits and vegetables consumption, price reductions are more effective than income policies. However, to achieve the recommended daily intake, poorer families should face a $55 \%$ price decrease.
\end{abstract}


Key words: Demand. QUAIDS Model. Fruits and vegetables.

JEL: D12, R22, C3

\section{Introdução}

O consumo insuficiente de frutas e hortaliças é considerado como um dos principais fatores de risco à incidência de doenças crônicas não transmissíveis (DCNTs). Estima-se que 20\% dos casos de câncer e 30\% das doenças cardíacas estão relacionados ao consumo irregular desses alimentos. Além disso, a sua substituição crescente por alimentos ricos em gorduras e açucares contribui para o sobrepeso (OMS, 2002). Como principal causa, os custos de alimentos mais saudáveis são barreiras a possíveis melhorias na dieta, especialmente em famílias de baixa renda. Em geral, alimentos mais calóricos e menos nutritivos são mais baratos e menos sensíveis à variação dos preços, ao contrário de alimentos com alto teor nutricional (Drewnowski et al., 2004; Claro; Monteiro, 2010).

Diante dos riscos à saúde associados a uma dieta inadequada e a importância do consumo de frutas e hortaliças, alguns estudos internacionais buscaram compreender os principais fatores associados à sua demanda (Feng; Chern, 2000; Mutuc et al., 2007; Bertail; Caillavet, 2008; Durham E Eales, 2010). Já no Brasil, existem poucas evidências sobre quais fatores são importantes para explicar e estimular seu consumo, concentradas na análise de grupos agregados ou itens básicos sem considerar a importância de alguns alimentos específicos na dieta dos brasileiros Coelho et al., 2010; Barbosa et al., 2010; Lucinda. Leifert, 2012).

O inquérito da POF de 2008/2009 evidencia a preferência dos brasileiros por alimentos pouco saudáveis. O consumo de frutas e hortaliças, por exemplo, equivale conjuntamente a um terço da quantidade recomendada pelo Mistério da Saúde, em média (Brasi, 2005; IBGE, 2010a). Como os preços desses alimentos são um dos principais limitadores a sua aquisição, este estudo pretende analisar como subsídios podem ser eficazes para que os brasileiros adquiram as quantidades adequadas. Além disso, pretende-se verificar possíveis diferenças entre níveis de rendimento, contribuindo para o direcionamento de ações específicas para cada realidade.

O artigo é estruturado em quatro seções, incluindo esta introdução. Na pró- 
xima seção é apresentada a forma funcional para a estimação dos sistemas de demanda, bem como os procedimentos econométricos e a base de dados. Em seguida, são discutidos os principais resultados. Na quarta seção, apresentam-se as considerações finais sobre a pesquisa.

\section{Referencial Analítico}

\subsection{Forma Funcional e procedimentos econométricos}

Para estimar o sistema de demanda de frutas e hortaliças, utiliza-se o modelo QUAIDS (Quadratic Almost Ideal Demand System), de Banks et al. (1997). Como se propõe uma análise desagregada, que resulta em um elevado número de observações com consumo zero, aplica-se o procedimento de estimação em dois estágios de Shonkwiller e Yen (1999). No primeiro estágio, estima-se um modelo de escolha binária (Probit), para cada equação separadamente, seguido pela estimação do sistema por SUR (regressões aparentemente não correlacionadas).

Justifica-se o uso desse procedimento, uma vez que dos 25 produtos analisa$\operatorname{dos}^{1}, 17$ não são consumidos em pelo menos $88 \%$ dos domicílios, independente da classe de rendimento. Até mesmo os bens mais populares (Banana, Tomate e Batata) tiveram baixa frequência de consumo. Como se tratam de produtos perecíveis o consumo zero parece decorrer do nível de desagregação na cesta de bens, os quais não fazem parte das preferências e possibilidades de compra da grande maioria dos domicílios.

Além de considerar os preços e dispêndio, o sistema de demanda estimado também inclui outras variáveis que captam a heterogeneidade dos consumidores (Pollak; Wales, 1981; Hovhannisyan; Gould, 2011). Dessa forma, o modelo estimado é o seguinte:

$$
\mathrm{w}_{\mathrm{ik}}=\phi\left(\mathrm{z}_{\mathrm{ik}}^{\prime} \hat{\alpha}\right)\left[\sum_{\mathrm{k}} \theta_{\mathrm{ik}} \mathrm{D}_{\mathrm{k}}+\alpha_{\mathrm{i}}+\sum_{\mathrm{j}} \gamma_{\mathrm{ij}} \log \mathrm{p}_{\mathrm{j}}+\beta_{\mathrm{i}} \log \left(\frac{\mathrm{m}_{\mathrm{r}}}{\mathrm{a}(\mathrm{p})}\right)+\frac{\lambda_{\mathrm{i}}}{\mathrm{b}(\mathrm{p})}\left\{\log \left(\frac{\mathrm{m}_{\mathrm{r}}}{\mathrm{a}(\mathrm{p})}\right)\right\}^{2}+\mathrm{u}_{\mathrm{i}} \hat{\mathrm{v}}_{\mathrm{k}}\right]+\varphi_{\mathrm{i}} \phi\left(\mathrm{z}_{\mathrm{ik}}^{\prime} \hat{\alpha}_{\mathrm{l}}\right)+\mathrm{V}_{\mathrm{ik}}
$$

em que, ${ }^{{ }^{\mathrm{k}}=} \frac{\mathrm{p}_{\mathrm{i}} \mathrm{q}_{\mathrm{i}}}{\sum_{\mathrm{i}=\mathrm{i}}^{n} \mathrm{p}_{\mathrm{i}}}$ é a parcela de gastos com o $i$-ésimo bem pelo $k$-ésimo do1 Ver produtos escolhidos na Tabela 2. 
micílio; $\log \mathrm{p}_{\mathrm{j}}$ é o logaritmo do preço do $j$-ésimo bem; $\mathrm{m}_{\mathrm{r}}$ é o dispêndio total com os produtos analisados; $\phi\left(\mathrm{z}_{\mathrm{ik}} \hat{\alpha}_{1}\right)$ e $\Phi\left(\mathrm{z}_{\mathrm{ik}} \hat{\alpha}_{1}\right)$ são as funções de densidade de probabilidade e de distribuição acumulada, respectivamente, obtidas no $1^{\circ}$ estágio do procedimento de Shonkwiller e Yen; a(p) é um índice de preços de Laspeyres; $\lambda_{\mathrm{i}}$ é o parâmetro requerido para o termo quadrático do dispêndio e o termo $\mathrm{b}(\mathrm{p})=\prod_{\mathrm{k}} \mathrm{p}_{\mathrm{k}}^{\beta_{\mathrm{k}}}$ é um agregador de preços Cobb-Douglas, requerido para manter a integrabilidade do termo quadrático; $D_{\mathrm{k}}$ é um vetor de variáveis que caracterizam o $k$-ésimo domicílio e $\theta_{\mathrm{ik}}$ são os parâmetros estimados para cada variável; $\alpha_{\mathrm{i}}, \beta_{\mathrm{i}}, \varphi_{\mathrm{i}}, \mathrm{u}_{\mathrm{i}}$ são parâmetros do modelo; $\mathrm{V}_{\mathrm{ik}}$ é o termo de erro.

Como na Pesquisa de Orçamentos Familiares (POF) o preço $p_{j}$ do $j$-ésimo bem não é disponibilizado, ele será representado pelo valor unitário de cada bem, obtido pela razão entre o dispêndio e a quantidade consumida do $i$-ésimo bem pelo $k$-ésimo domicílio (Mutuc et al., 2007). Entretanto, a utilização de valores unitários pode comprometer a estimação da equação de demanda, pois é comum que eles não sejam exógenos, incorporando atributos como qualidade do bem. Para solucionar este problema, aplica-se o método de Cox e Wohlgenant (1986), que consiste em ajustar os valores unitários para diferenças de qualidade. Assume-se que os desvios em relação aos valores unitários médios refletem "efeitos de qualidade" induzidos pelas características domiciliares, como também fatores não sistemáticos ligados à oferta ${ }^{2}$.

A variável $\hat{\mathrm{v}}_{\mathrm{k}}$ é utilizada para corrigir o problema da endogeneidade do dispêndio. Esse problema ocorre porque, ao considerar o dispêndio com frutas e hortaliças como fracamente separável ${ }^{3}$, assume-se que a quantidade demandada do $i$-ésimo bem considerado $\left(\mathrm{q}_{\mathrm{i}}\right)$ é uma função apenas dos preços e do dispêndio com frutas e hortaliças $\left(\mathrm{m}_{\mathrm{r}}\right)$, sendo que esta depende do dispêndio total. Para corrigir este problema, foi usada a abordagem de estimação por regressão aumentada de Blundell e Robin (1999). Esta abordagem consiste em duas etapas: na primeira, o dispêndio total é regredido num conjunto de variáveis exógenas, incluindo aquelas que podem influenciar diretamente as parcelas de gasto, considerando a renda per capita como instrumento. $\mathrm{Na}$ segunda etapa, os resíduos $\left(\hat{\mathrm{v}}_{\mathrm{k}}\right)$, obtidos nessa estimação, são incluídos como variável explicativa nas equações das parcelas de gasto (1), conjuntamente com o dispêndio total com os bens de interesse (Blundell e Robin, 1999).

Para encontrar as elasticidades-preço próprias e cruzadas, primeiramente, diferencia-se a equação (1) em relação ao logaritmo do dispêndio e dos preços, respectivamente (Banks et al. , 1997). elasticidades-preço marshallianas $\left(\mathrm{e}_{\mathrm{ij}}^{\mathrm{u}}\right)$ ( podem ser escritas como:

$$
\mathrm{e}_{\mathrm{ij}}^{\mathrm{u}}=\frac{\mu_{\mathrm{ij}}}{\mathrm{w}_{\mathrm{i}}}-\delta_{\mathrm{ij}}
$$

2 Para mais detalhes sobre o método, ver Cox e Wohlgenant (1986) e Deaton (1988). 3 Ver Deaton e Muellbauer (1980a), pags. 127-128. 
em que $\mu_{\mathrm{ij}}$ são as derivadas de $\mathrm{w}_{\mathrm{i}}$ em relação ao logaritmo do preço do j-ésimo bem, respectivamente e $\delta_{\mathrm{ij}}$ é o Delta Kronecker, o qual assume os valores 1, se $\mathrm{i}=\mathrm{j}$ e $\mathrm{o}$, caso contrário.

Recorre-se a rotina de programação descrita em Poi (2008), devido à flexibilidade em se adaptar a qualquer especificação, bem como por permitir a utilização de qualquer índice de preço. Nesta, as restrições de Simetria e Homogeneidade são impostas ${ }^{4}$ na programação dos parâmetros. Acrescentaram-se o procedimento de Shonkwiller e Yen, variáveis de controle e correção da endogeneidade do dispêndio. Para garantir a imposição de aditividade das parcelas de gasto, tratou-se um dos bens como "residual" e estimou-se o sistema de demanda para $n-1$ bens (Yen et al., 2003). O bem residual escolhido foi o Brócolis, pois tem menor participação nos gastos com a cesta de bens escolhida. A restrição de aditividade permitiu a obtençãodos parâmetros para o Brócolis e suas elasticidades.

Estimou-se o QUAIDS como um sistema não linear, devido à função $b(p)$. .

Em Poi (2008), são estimados apenas os parâmetros $\alpha, \beta, \gamma, \lambda$. Para a cesta de bens utilizada $(n=25)$, o total de parâmetros corresponderia a 372. Ao aplicar o procedimento de Shonkwiller e Yen, acrescentaram-se 24 parâmetros $\varphi$, cada um nas $n$-1 equações. Incluíram-se também os parâmetros de correção da endogeneidade $\mathrm{u}$ e das variáveis demográficas $\theta$. A quantidade final de parâmetros estimados foi de 756 parâmetros.

\subsection{Cálculo dos efeitos das mudanças nos preços de frutas e hortaliças}

A partir dos parâmetros estimados no sistema de demanda, calculam-se as elasticidades-preços, expressas em (2). Ao considerar o efeito da redução de preços de todos os produtos, a mudança da quantidade consumida deve considerar, além das elasticidades-próprias, as elasticidades-preço cruzadas, devido às relações de complementaridade e substituição entre os bens. Logo, a variação da quantidade após a intervenção nos preços é dada pelo seguinte produto matricial (Leifert; Lucinda, 2012):

$$
\Delta \mathrm{Q}=\left(\mathrm{E}^{*} \Delta \mathrm{P}^{\prime}\right)^{\prime}
$$

em que $\Delta \mathrm{Q}$ é um vetor com as $n$ variações percentuais das quantidades pelo 4 Devido a limitações computacionais, as restrições foram apenas impostas, não testadas, uma vez que para o modelo sem simetria e sem homogeneidade, seria necessário estimar 1057 parâmetros. A quantidade excessiva de parâmetros dificultou a convergência dos resultados. 
domicílios depois da política; $E$ é uma matriz nxn das elasticidades-preço próprias e cruzadas do $i$-ésimo bem; $\Delta \mathrm{P}$ contém a variação dos preços para os $n$ bens, dada por $\left(\tau \%{ }^{*} \mathrm{p}_{\mathrm{o}}\right)$, sendo $\tau \%$ o valor percentual descontado. A quantidade consumida de frutas e hortaliças é dada pelo somatório da quantidade média consumida de todos os produtos depois da mudança nos preços

Como as quantidades consumidas estão agregadas em nível domiciliar, pondera-se pelo total de pessoas nos domicílios, por meio da escala de adultos equivalentes (AE). Utiliza-se a medida AE exposta em Rocha (1990), a qual considera que a participação na aquisição de cada membro no domicílio é contada como alguma fração de um adulto do sexo masculino, sendo o tamanho dos domicílios a soma dessas frações. As ponderações são apresentadas na Tabela 1.

TABELA 1 - FRAÇÕES DE ADULTO EQUIVALENTE POR IDADE E SEXO

\begin{tabular}{c|c|c|c|c|c|c|c|c|c}
\hline \multirow{2}{*}{ Sexo } & \multicolumn{10}{c}{ Idade } \\
\cline { 2 - 9 } & $<1$ & 1 a 3 & 4 a 6 & 7 a 9 & 10 a 13 & 14 a 17 & 18 a 30 & 31 a 60 & $>60$ \\
\hline Homem & 0,269 & 0,494 & 0,64 & 0,736 & 0,812 & 0,974 & 1 & 1,001 & 0,843 \\
Mulher & 0,249 & 0,461 & 0,577 & 0,65 & 0,717 & 0,762 & 0,721 & 0,739 & 0,678 \\
\hline
\end{tabular}

Fonte: Rocha (1998).

\subsection{Base de dados}

As informações utilizadas nesse estudo são provenientes dos microdados da Pesquisa de Orçamentos Familiares (IBGE, 2010b), pesquisa de caráter amostral, realizada pelo Instituto Brasileiro de Geografia e Estatística (IBGE), para os anos 2008/2009. Essa pesquisa, realizada para uma amostra de 55.970 domicílios, situados nas áreas urbanas e rurais de todo território brasileiro, disponibiliza informações sobre a composição orçamentária doméstica e sobre as condições de vida da população, visando mensurar as estruturas de consumo, dos gastos e fontes de rendimento. Além disso, a pesquisa mostra informações antropométricas, bem como o consumo e despesa individual (IBGE, 2010c).

Os produtos considerados no sistema de demanda neste trabalho foram escolhidos com base na classificação dos grupos alimentares da POF, devido a sua importância na aquisição domiciliar em relação aos demais itens dentro do subgrupo alimentar analisado, como também a sua importância para uma alimentação saudável e variada (IBGE, 2010a). Os 25 bens escolhidos, apre- 
sentados na Tabela 2, correspondem a aproximadamente $80 \%$ da quantidade domiciliar per capita de todos os tipos de frutas e hortaliças considerados pela POF, permitindo obter um sistema representativo para o grupo alimentar em análise, sem criar um número excessivo de equações.

TABELA 2 - PROPORÇÃO DE DOMICÍLIOS COM CONSUMO ZERO POR FRUTAS E HORTALIÇAS SELECIONADAS, 2009

\begin{tabular}{|c|c|c|c|}
\hline \multirow{2}{*}{ Produtos } & \multicolumn{3}{|c|}{ Classes de rendimento per capita* } \\
\hline & Inferior & Intermediária & Superior \\
\hline \multicolumn{4}{|c|}{ Frutas de clima tropical } \\
\hline Abacaxi & $96,2 \%$ & $93,4 \%$ & $89,8 \%$ \\
\hline Banana & $62,2 \%$ & $58,1 \%$ & $49,2 \%$ \\
\hline Laranja & $79,5 \%$ & $74,6 \%$ & $67,3 \%$ \\
\hline Limão & $95,2 \%$ & $93,8 \%$ & $88,9 \%$ \\
\hline Mamão & $94,4 \%$ & $89,6 \%$ & $77,9 \%$ \\
\hline Manga & $93,8 \%$ & $93,2 \%$ & $91,5 \%$ \\
\hline Melão & $98,7 \%$ & $97,5 \%$ & $94,8 \%$ \\
\hline Melancia & $91,5 \%$ & $90,9 \%$ & $89,7 \%$ \\
\hline Tangerina & $96,7 \%$ & $94,0 \%$ & $89,6 \%$ \\
\hline \multicolumn{4}{|c|}{ Frutas de clima temperado } \\
\hline Maçã & $83,9 \%$ & $78,7 \%$ & $69,3 \%$ \\
\hline Morango & $99,4 \%$ & $98,1 \%$ & $95,2 \%$ \\
\hline Pera & $99,1 \%$ & $97,3 \%$ & $91,6 \%$ \\
\hline Uva & $96,0 \%$ & $93,2 \%$ & $88,7 \%$ \\
\hline \multicolumn{4}{|c|}{ Hortaliças Folhosas } \\
\hline Alface & $84,6 \%$ & $73,3 \%$ & $65,3 \%$ \\
\hline Brócolis & $99,6 \%$ & $98,6 \%$ & $94,7 \%$ \\
\hline Couve & $95,5 \%$ & $92,8 \%$ & $90,1 \%$ \\
\hline Repolho & $93,0 \%$ & $90,3 \%$ & $88,3 \%$ \\
\hline \multicolumn{4}{|c|}{ Hortaliças Frutosas } \\
\hline Abóbora & $91,3 \%$ & $90,8 \%$ & $89,6 \%$ \\
\hline Chuchu & $94,8 \%$ & $92,6 \%$ & $89,9 \%$ \\
\hline Pepino & $96,0 \%$ & $94,2 \%$ & $91,7 \%$ \\
\hline Tomate & $48,4 \%$ & $50,8 \%$ & $49,8 \%$ \\
\hline (Continua) & & & \\
\hline
\end{tabular}




\begin{tabular}{|c|c|c|c|}
\hline \multicolumn{4}{|c|}{ (Continuação) } \\
\hline \multirow{2}{*}{ Produtos } & \multicolumn{3}{|c|}{ Classes de rendimento per capita* } \\
\hline & Inferior & Intermediária & Superior \\
\hline \multicolumn{4}{|c|}{ Hortaliças Tuberosas } \\
\hline $\begin{array}{l}\text { Batata- } \\
\text { inglesa }\end{array}$ & $75,1 \%$ & $72,2 \%$ & $71,4 \%$ \\
\hline Beterraba & $95,7 \%$ & $93,7 \%$ & $91,5 \%$ \\
\hline Cenoura & $85,9 \%$ & $81,4 \%$ & $75,1 \%$ \\
\hline Mandioca & $89,9 \%$ & $89,2 \%$ & $89,8 \%$ \\
\hline
\end{tabular}

Fonte: Resultados da pesquisa.

* As classes de rendimento foram criadas com base na distribuição da renda domiciliar per capita dos domicílios considerados nas estimações. Ver Tabela 2.

Dos 55.970 domicílios entrevistados pela POF, 1.199 não informaram alguma das variáveis demográficas consideradas. Também foram retiradas 116 observações, cujos preços finais, obtidos após a estimação pelo procedimento de Cox e Wohlgenant (1986), possuíam valores muito discrepantes. Ressalta-se que alguns domicílios declararam o consumo de banana, porém não declararam despesa. Esses casos não foram retirados e os preços imputados equivalem ao preço médio da UF. A amostra final foi de 28.128 observações, correspondente aos domicílios que declararam o consumo de pelo menos um dos bens (incluindo o bem residual).

Para permitir uma análise por faixas de renda, os domicílios foram divididos de acordo com quartos da distribuição de renda per capita, devido às possíveis discrepâncias de consumo. O primeiro grupo (rendimento inferior) pertence ao $1^{\mathrm{O}}$ quartil da distribuição de renda domiciliar per capita, que inclui domicílios com renda até $\mathrm{R} \$ 285,02 /$ pessoa. No segundo grupo (rendimento intermediário) estão os domicílios com renda per capita entre de 285,02 e $984,45\left(2^{\circ} \mathrm{e} 3^{\circ}\right)$. No grupo com rendimento mais alto estão os domicílios do $4^{\circ}$ quartil da distribuição, cuja renda per capita é superior a $R \$ 984,45$ (Tabela 3 ). 
SILVA, M. M. da C.; COELHO, A. B. A Influência dos Preços sobre a Demanda Domiciliar por Frutas e Hortaliças:...

TABELA 3 - INTERVALOS DE RENDA PER CAPITA CONSIDERADOS PARA A CLASSIFICAÇÃO DAS CLASSES DE RENDIMENTO

\begin{tabular}{lcc}
\hline Quartil & Classe & $\begin{array}{c}\text { Intervalo de renda per } \\
\text { capita }\end{array}$ \\
\hline $1^{\mathrm{o}}$ & Inferior & Até $\mathrm{R} \$ 285,02 /$ pessoa \\
$2^{\mathrm{o}}$ e $3^{\mathrm{o}}$ & Intermediária & $\begin{array}{c}\text { de } \mathrm{R} \$ 285,03 \text { e até } \mathrm{R} \$ \\
984,45 / \text { pessoa }\end{array}$ \\
$4^{\mathrm{o}}$ & Superior & maior que $\mathrm{R} \$ 984,45 /$ \\
& & pessoa \\
\hline
\end{tabular}

Fonte: Resultados da Pesquisa.

De forma geral, o Guia Alimentar elaborado pelo Ministério da Saúde, com base na Organização Mundial de Saúde (OMS), sugere que o consumo mínimo de Frutas e Hortaliças deveria corresponder a 400 gramas por dia. No entanto, a publicação da POF 2008/2009 sobre a aquisição per capita dos brasileiros mostra que o consumo diário desses alimentos pelos brasileiros corresponde a cerca de um terço do recomendado. Além disso, nota-se como a variabilidade desse tipo de alimento é baixa, visto que a participação na quantidade está concentrada em frutas mais populares, independente da classe de rendimento a qual o domicílio pertence (IBGE, 2010a).

A Tabela $4^{5}$ compara a quantidade diária per capita (medida usada pelo IBGE) e por AE em todos os domicílios entrevistados pela POF e nos domicílios considerados nas estimações (domicílios que consomem pelo menos um dos bens da cesta). Verifica-se o quão baixo está a aquisição domiciliar média em relação à quantidade recomendada pelo Guia, tanto em termos per capita, quanto em AE, considerando todos os domicílios entrevistados. Com relação à amostra considerada nas estimações, a Tabela 4 mostra que menos da metade dos domicílios que adquiriram a quantidade recomendada de frutas e hortaliças. A média de consumo diário/AE e per capita corresponde a $320 \mathrm{~g}$ e $267 \mathrm{~g}$, respectivamente, sendo que apenas $32 \%$ dos domicílios alcançaram a quantidade recomendada. Na classe de renda inferior, o consumo cai para 173 g/AEe 140 g per capita, em que menos de $15 \%$ dos indivíduos adquiriram 320 g/AE. Apesar de ter média de consumo diário acima de 320 , cerca de metade dos domicílios classe de renda superior adquiriram quantidades maiores do que o recomendado. Embora a disparidade entre classes seja evidente, o baixo consumo está presente em todos os níveis de rendimento.

5 Nesta tabela, apresenta-se o consumo médio dos produtos utilizados nas estimações, os quais correspondem a $80 \%$ do consumo em $\mathrm{Kg}$ /dia de frutas e hortaliças. Pode-se dizer que esses produtos deveriam corresponder em média a $320 \mathrm{~g}$ da quantidade diária recomendada. 
TABELA 4 - QUANTIDADE MÉDIA EM KG/DIA ADQUIRIDOS POR AE E PER CAPITA, BRASIL E CLASSES DE RENDA, 2009

\begin{tabular}{lcccc}
\hline & Brasil & Inferior & Intermediária & Superior \\
\hline & \multicolumn{3}{c}{ Total } & \\
\hline $\begin{array}{l}\text { Kg por AE / dia } \\
\text { Kg per capita/ dia }\end{array}$ & 0,168 & 0,078 & 0,162 & 0,300 \\
$\begin{array}{l}\text { Kg por AE / } \\
\text { dia }>=3209\end{array}$ & $17 \%$ & $6 \% 061$ & 0,129 & 0,241 \\
$\begin{array}{l}\text { Kg per capita/ } \\
\text { dia }>=320 g\end{array}$ & $13 \%$ & $4 \%$ & $17 \%$ & $31 \%$ \\
\hline $\begin{array}{l}\text { Kg por AE / dia } \\
\text { Kg per capita/ dia }\end{array}$ & 0,320 & 0,173 & $13 \%$ & $26 \%$ \\
$\begin{array}{l}\text { Kg por AE / } \\
\text { dia }>=320 g\end{array}$ & $32 \%$ & $14 \%$ & 0,305 & 0,495 \\
$\begin{array}{l}\text { Kg per capita/ } \\
\text { dia }>=320 \text { Amostra }\end{array}$ & $27 \%$ & $10 \%$ & 0,254 & 0,420 \\
\hline
\end{tabular}

Fonte: Resultados da pesquisa.

\section{Resultados e Discussão}

Como já mencionado, foram estimados 756 parâmetros no sistema de 24 equações ( $n-1$ produtos). Desses, $36 \%$ foram significativos no sistema estimado para a classe inferior, $44 \%$ na classe intermediária e $32 \%$ na classe superior ${ }^{6}$. Antes de analisar as elasticidades, verifica-se o comportamento dos parâmetros $\lambda$, utilizados para expressar a forma quadrática do dispêndio nas equações de demanda e $\mathrm{u}$, usados para corrigir a endogeneidade do dispêndio, respectivamente. Os parâmetros $\mathbf{u}_{\mathrm{i}}$ referem-se aos resíduos estimados da forma reduzida do dispêndio, incluídos no $2^{\circ}$ estágio para testar e controlar o problema da endogeneidade do dispêndio total com os $n$ bens. A não significância de $u$ implica a rejeição da hipótese de endogeneidade na $i$-ésima equação de demanda. Ao contrário, se u for significativo, pode-se dizer que ao considerar a exogeneidade do dispêndio total nas equações de demanda por frutas e hortaliças, geram-se estimativas inconsistentes (Blundell; Robin, 1999).

A partir dos coeficientes estimados dos sistemas de demanda, pode-se encon6 Por questões de espaço, esses resultados não serão apresentados e sim apenas as elasticidades calculadas a partir deles. Os resultados completos estão disponíveis sob requisição aos autores e em ??? (suprimido para não identificar os autores do artigo) 
trar o grau de sensibilidade dos consumidores em relação às variações dos preços elasticidades-preço marshallianas da demanda para os 25 produtos nas três classes de rendimento são apresentadas na Tabela 5, considerando-se os pontos médios da amostra. Por meio do método delta, foram encontrados os desvios-padrões, permitindo-se fazer inferência estatística sobre os resultados. Verifica-se que as relações encontradas são estatisticamente significativas para a maioria dos bens, nas três classes, considerando um nível de significância de $10 \%$.

Os bens analisados possuem elasticidade-preço própria negativa (exceto Pera, Melão e Brócolis, na classe inferior), com predominância de bens com demanda elástica em todas as classes de rendimento. O comportamento é semelhante entre a classe inferior e intermediária, principalmente para as frutas. Verifica-se que, dos produtos analisados, 10 tiveram maiores elasticidades-preço nos domicílios da amostra que estão na classe inferior, principalmente em alguns produtos pertencentes às Frutas tropicais, Frutas de clima temperado e Hortaliças Tuberosas. A classe intermediária apresentou grau de sensibilidade mais elevado em sete produtos, sendo a maioria pertencente às Frutas tropicais e Hortaliças Frutosas. Logo, a hipótese que as classes com menor nível de rendimento domiciliar são mais sensíveis às variações nos preços de cada bem não é rejeitada, uma vez que os consumidores mais pobres são os que mais são impactados pelas variações dos preços dos itens da cesta considerada, seguido daqueles com nível de renda intermediário.

TABELA 5 - ELASTICIDADES-PREÇO MARSHALLIANAS , POR CLASSES DE RENDIMENTO, 2009

\begin{tabular}{clccc}
\hline Produtos & Inferior & Intermediária & Superior \\
\hline \multicolumn{4}{c}{ Frutas de clima tropical } \\
\hline Abacaxi & $-2,688^{* *}$ & $-1,931^{* *}$ & $-0,746^{* *}$ \\
Banana & $-0,669^{* *}$ & $-0,72^{* *}$ & $-0,798^{* *}$ \\
Laranja & $-1,685^{* *}$ & $-1,472^{* *}$ & $-1,026^{* *}$ \\
Limão & $-4,298^{* *}$ & $-2,454^{* *}$ & $-1,368^{* *}$ \\
Mamão & $-0,384$ & $-1,496^{* *}$ & $-1,024^{* *}$ \\
Manga & $-0,186$ & $-1,805^{* *}$ & $-1,544^{* *}$ \\
Melancia & $-2,398^{* *}$ & $-2,336^{* *}$ & $-1,610^{* *}$ \\
Melão & 1,010 & $-4,616^{* *}$ & $-0,903^{* *}$ \\
Tangerina & $-0,723^{*}$ & $-2,965^{* *}$ & $-1,248^{* *}$ \\
\hline \multicolumn{5}{c}{ Frutas de clima temperado } \\
\hline Maçã & $-2,225^{* *}$ \\
\hline
\end{tabular}




\begin{tabular}{cccc}
\hline (Continuação) & \multicolumn{3}{c}{} \\
\hline Morango & $-0,130$ & $-0,87^{* *}$ & $-1,518^{* *}$ \\
Pera & $0,891^{* *}$ & $-3,081^{* *}$ & $-1,677^{* *}$ \\
Uva & $-3,153^{* *}$ & $-2,037^{* *}$ & $-1,476^{* *}$ \\
\hline Hortaliças Folhosas \\
\hline Blface & $-1,42^{* *}$ & $-1,355^{* *}$ & $-1,394^{* *}$ \\
Brócolis & $3,014^{*}$ & $-1,212$ & $-1,602$ \\
Couve & $-2,035^{* *}$ & $-2,184^{* *}$ & $-2,345^{* *}$ \\
Repolho & $-0,768^{* *}$ & $-1,290^{* *}$ & $-1,335^{* *}$ \\
\hline Abóbora & Hortaliças Frutosas & $-2,060^{* *}$ \\
Chuchu & $-3,362^{* *}$ & $-2,283^{* *}$ & $-1,294^{* *}$ \\
Pepino & $-1,001^{* *}$ & $-2,055^{* *}$ & $-1,299^{* *}$ \\
Tomate & $-1,546^{* *}$ & $-2,211^{* *}$ & $-0,924^{* *}$ \\
\hline Batata & $-1,041^{* *}$ & $-1,030^{* *}$ & $-1,335^{* *}$ \\
Cenoura & Hortaliças Tuberosas & $-2,192^{* *}$ \\
Beterraba & $-1,709^{* *}$ & $-1,564^{* *}$ & $-1,282^{* *}$ \\
Mandioca & $-1,847^{* *}$ & $-1,669^{* *}$ & $-1,555^{* *}$ \\
\hline & $-1,842^{* *}$ & $-2,293^{* *}$ & \\
\hline
\end{tabular}

Nível de significância: * $10 \%,{ }^{* *} 5 \%$.

Fonte: Resultados da pesquisa.

Em geral, os domicílios brasileiros mostraram-se sensíveis às variações nos preços de cada produto, independente da classe de renda. Assim, de acordo com a amostra considerada, para incentivar o consumo de frutas e hortaliças, e consequentemente, a melhoria dos hábitos alimentares, subsidiar tais produtos pode ser eficaz. Isso corrobora as considerações de Sarti et al. (2011), o qual sintetiza que mudanças no preço podem ter maior contribuição sobre a demanda.

Para efeito de comparação, em Leifert e Lucinda (2012), as elasticidades-dispêndio encontradas para os agregados 'Frutas' e 'Verduras e Legumes' foram de, respectivamente, 0,968 e 0,904, e elasticidades-preço próprias de $-0,816$ e -0,848. Nota-se que a agregação nas categorias Frutas e Verduras e Legumes gerou valores de elasticidade-preço abaixo de um, classificando as demandas dessas categorias como inelásticas. Provavelmente, a agregação faz com que haja perda de informações importantes sobre o real comportamento dos consumidores em relação a cada bem. O mesmo ocorre em Claro e Monteiro (2010), o qual apresentou uma relação inelástica entre a aquisição 
calórica e o preço de frutas e hortaliças por cada $1000 \mathrm{kcal}$. Em muitos casos, a aquisição física e calórica tem predominância de produtos mais básicos. Como visto na Tabela 6 , a maioria dos bens são classificados como elásticos, sendo inelásticos os bens Banana, Batata e Tomate, que são bens mais frequentes na alimentação dos brasileiros.

Além do seu próprio preço, a demanda pode ser influenciada pelos preços de outros bens. Devido à suposição de separabilidade fraca, as comparações são feitas apenas entre os bens considerados. Os bens podem ser classificados, de acordo com as elasticidades-preço marshallianas, como complementares brutos quando o aumento do preço do $j$-ésimo bem reduz a quantidade demandada do $i$-ésimo bem e substitutos brutos quando esse aumento eleva a quantidade demandada. Para facilitar a visualização dessas relações, nas Tabelas A1, A2 e A3 do Apêndice, classificam-se com C os bens complementares, e S, quando substitutos. Consideram-se apenas as relações estatisticamente significativas a no máximo $10 \%$.

Na Tabela A1, são apresentadas as relações de complementaridade e substituição para os domicílios da amostra situados na faixa inferior de renda per capita. Foram captadas 204 relações estatisticamente significativas, sendo que 110 são de substituição entre os bens, e 94 de complementaridade. Para a classe intermediária (Tabela A2), das 236 relações, 141 são de substituição e 95 de complementaridade. Na classe superior (Tabela A3), os preços dos outros bens têm menos influência significativa na aquisição de determinado bem, em comparação aos demais domicílios. Foram encontradas 68 relações de substituição e 61 de complementaridade, totalizando 129 relações significativas. Dado que a maioria das relações significativas foi de substituição, evidencia-se que a demanda por alimentos saudáveis sofre influência da variação nos seus preços relativos, principalmente em domicílios com renda baixa e intermediária.

A relação de complementaridade entre bens indica um consumo estreitamente vinculado, enquanto que bens substitutos são competitivos, sendo essas relações importantes para a eficácia de políticas que objetivam melhorar a alimentação (SARTI, et al. 2011). Em todas as classes, o consumo concorrente aparece com mais frequência nas hortaliças, pois têm mais casos de substituição entre si e entre quase todas as frutas. Estas, por sua vez, são complementares entre si, principalmente as pertencentes às Frutas tropicais. Nos domicílios pobres, verifica-se que todos os itens pertencentes à categoria Frutas de clima Temperado, que tiveram relações significativas, são substitutas. O mesmo ocorre na categoria Hortaliças Tuberosas, em domicílios mais ricos, cujo consumo concorrente foi captado apenas entre Beterraba e Mandioca.

Na Tabela A4 do Apêndice, apresentam-se os efeitos de um desconto de 5\% para cada item. Vale salientar que a análise é parcial e só permite visualizar os efeitos do lado demanda, não permitindo discutir os efeitos sobre a oferta e o equilíbrio geral da economia. Assim como em Cash et al. (2005), pressupõe- 
-se que os gastos são assumidos pelo governo, sem levar em considerações possíveis distorções no mercado ${ }^{7}$.

Nos domicílios pobres, a quantidade final demandada por AE passaria a ser equivalente a $187 \mathrm{~g}$ com a redução nos preços, que embora abaixo do recomendado ${ }^{8}$, representa um aumento de $8 \%$. Destacam-se os impactos sobre os bens Melão e Pera, cujo aumento na quantidade final é muito expressiva, comparado aos demais bens, mesmo estes bens apresentando demanda inelástica. É importante salientar que a variação elevada parte de uma quantidade inicial muito pequena. No caso da Pera, por exemplo, a quantidade consumida aumenta em 4,5 vezes (de $0,4 \mathrm{~g} / \mathrm{AE}$ para quase $2 \mathrm{~g} / \mathrm{AE}$ ). Entretanto, a quantidade final ainda é ínfima quando comparada com a quantidade (tanto inicial e final) adquirida desse bem nas demais classes. A razão para esse expressivo aumento deve-se às relações de complementaridade com outros bens.

Em alguns produtos (Abacaxi, Limão, Melancia, Tangerina, Morango, Abobora, Chuchu, Beterraba e Mandioca), a redução de preço seria ineficaz para aumentar o consumo, uma vez que reduz a quantidade consumida de cada um desses bens. Mesmo que alguns desses bens apresentem demanda elástica, as relações de substituição com outros bens podem ser responsáveis por esse resultado, uma vez que, dado a redução no preço de todos os bens, para manter o dispêndio total, os consumidores irão preferir os substitutos desses bens, de acordo com seu grau de sensibilidade.

O efeito final de uma redução nos preços de $5 \%$ é suficiente para elevar a aquisição diária por $\mathrm{AE}$ ao nível de consumo adequado (320 g/dia per capita) nos domicílios com nível de renda intermediário, cujo aumento foi de 8,7\%. A maior variação ocorre com a quantidade demandada de Pera (45\%), seguido pela Manga (35\%), Alface e Brócolis (31\% em ambos). Para esses domicílios, também há a queda na quantidade final de alguns bens (Melão, Melancia, Tangerina, Morango, Brócolis, Abobora, Pepino, Chuchu). Para a média de consumo em domicílios ricos, a redução de preços aparentemente não seria necessária, pois estes já consumiam 495 g/dia por AE. Mesmo assim, a quantidade média total aumenta em 10,6\%. Assim, não se pode afirmar que o efeito de tal medida seja mais eficaz em domicílios pobres. De fato, embora os domicílios com renda domiciliar inferior sejam mais sensíveis à modificação dos preços de cada bem individualmente, as relações de substituição, com elevadas elasticidades-preço cruzadas, não favorecem a aplicação de um desconto em todos os preços.

Mesmo existindo casos como os mencionados acima, a redução de preços é importante para elevar a quantidade total demandada. Entretanto, para que os domicílios pobres alcancem a quantidade diária recomendada, em média, é necessário que os preços caiam em mais de 50\%. Para uma melhor visualização dos efeitos dessa política, o Quadro 1 mostra os efeitos finais sobre a quan- 
tidade demandada em AE por subcategoria. Embora a queda na quantidade demandada ocorra em alguns bens, a variação de cada categoria é positiva. Destaque para a demanda domiciliar por Frutas de Clima Temperado, em domicílios mais pobres, cuja quantidade final aumenta de $9 \mathrm{~g} / \mathrm{AE}$ para $13 \mathrm{~g} /$ AE (aumento de 48\%) e as Hortaliças Folhosas, que aumenta de 7,8 g para 9,4 (21\%). Entretanto, tem-se um impacto pequeno sobre a quantidade demandada total por Hortaliças Frutosas e Tuberosas.

O elevado preço das Frutas de clima temperado e Hortaliças Folhosas pode ser uma das principais barreiras para a aquisição desses produtos, excluindo-os do conjunto de possibilidades dos consumidores. Assim, a redução dos preços contribuiria para que domicílios com renda baixa possam adquiri-los. A política só não é mais eficaz para aumentar a quantidade total, pois essa redução não é suficiente para incentivar os consumidores a adquirir alguns bens, principalmente àqueles pertencentes às Hortaliças Frutosas e Tuberosas, provavelmente pela elevada relação de substituição desses bens para com os demais da cesta analisada.

O impacto sobre a demanda por Frutas de clima Temperado em domicílios com nível de renda intermediário e superior também é elevado, cuja variação total corresponde a $25 \%$ e $23 \%$, respectivamente. Para as demais categorias, a redução de preços de $5 \%$ também é eficaz, visto que é capaz de elevar a quantidade final em um valor proporcionalmente maior, com exceção apenas para as Hortaliças Folhosas, cujo aumento equivale a $4 \%$.

QUADRO 1 - MUDANÇA PERCENTUAL NA QUANTIDADE DEMANDADA POR SUBCATEGORIA, DADO UMA REDUÇÃO DE 5\% NOS PREÇOS, CLASSES DE RENDIMENTO, 2009

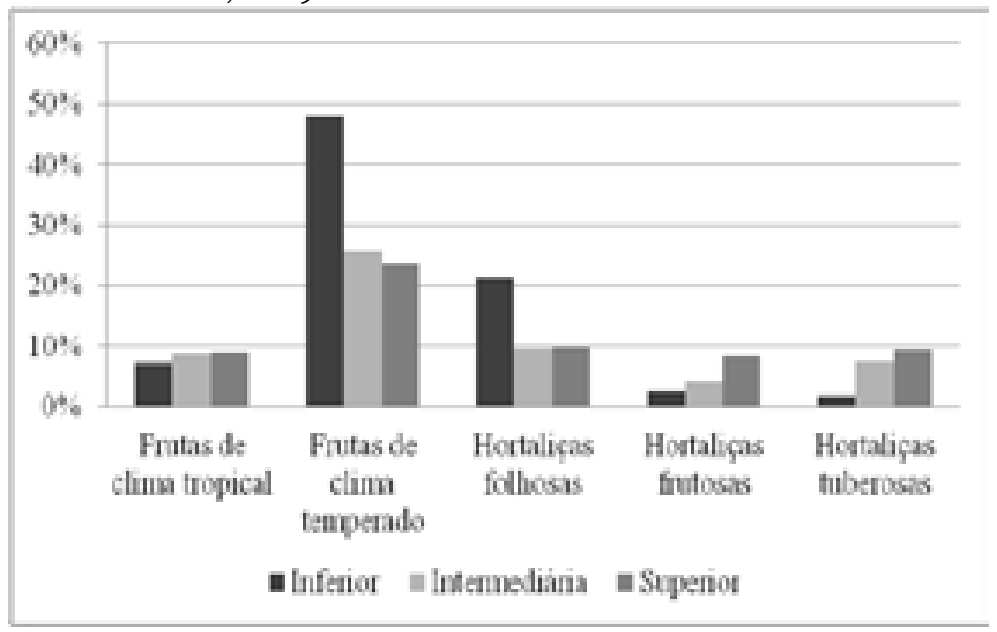

Fonte: Resultados da pesquisa. 
Em Cash et al. (2005), discute-se que a redução nos preços seria importante para reduzir as disparidades de consumo entre as classes de renda, uma vez que os consumidores norte-americanos mais pobres são mais sensíveis, sendo a intervenção mais eficaz para esses consumidores do que para aqueles consumidores mais ricos. De fato, a aquisição de frutas e hortaliças no Brasil é diferente entre domicílios pobres e ricos. Mesmo os domicílios considerados nas estimações (os quais deveriam ter dispêndio total positivo com pelo menos um dos bens da cesta), a quantidade média adquirida (em AE) nos domicílios pobres equivale a pouco mais de um terço do que é consumido nos domicílios mais ricos, e à metade do que é recomendado. Entretanto, os efeitos de uma redução em seus preços, apesar de ser eficaz em todas as classes, beneficiam ainda mais os domicílios com alta renda.

\section{Considerações Finais}

Uma das principais contribuições deste estudo para a literatura nacional sobre demanda por alimentos foi mostrar o comportamento de consumidores em diferentes classes de rendimento no processo de escolha de alimentos considerados saudáveis, optando-se por uma análise desagregada em 25 produtos pertencentes à categoria alimentar das frutas e hortaliças. De fato, foi verificado que a decisão de consumo difere entre produtos e níveis de renda, o que torna a análise agregada pouco informativa. É notável a discrepância de aquisição entre os domicílios pobres e ricos, sendo que a maioria dos produtos está fora do conjunto de possibilidades dos domicílios mais pobres, principalmente pelo seu alto valor e pela insuficiência de renda.

Ao simular uma redução de 5\% nos preços dos bens analisados pode-se constatar sua pouca eficácia sobre a demanda em domicílios mais pobres. Entretanto, verificou-se uma elevação expressiva na quantidade consumida de produtos pertencentes às frutas de clima temperado e hortaliças folhosas, os quais tem baixa participação no consumo desses domicílios. De forma geral, pode-se considerar que, caso o objetivo seja reduzir as disparidades de consumo entre as classes, o percentual de redução dos preços deve ser diferenciado entre as classes. Isto porque, para que a média de consumo dos indivíduos da classe intermediaria alcance a quantidade recomendada, a redução de $5 \%$ nos preços já seria eficaz. No entanto, para que isso seja alcançado pelos indivíduos com renda inferior, em média, a redução deveria ser equivalente a 55\%. Seria interessante analisar os efeitos dos subsídios, conjuntamente com políticas informativas e de transferências de renda. 
Apesar dos resultados encontrados possibilitarem um melhor entendimento dos fatores que podem contribuir para uma dieta mais saudável no Brasil, deve-se levar em consideração algumas limitações. Muitas destas limitações são devidas a estrutura dos dados utilizados, como por exemplo, as informações de consumo alimentar dentro do domicílio estão agregadas para todos os moradores. Uma análise de demanda individualizada poderia gerar resultados mais precisos, uma vez que seguir uma dieta saudável é uma decisão tomada pelo indivíduo, e não necessariamente, pelo responsável pelo domicílio. Além disso, seria interessante verificar a relação desses bens com alimentos pouco saudáveis. Outra limitação importante seria considerar o desenho amostral nas estimações, os quais podem ter efeitos sobre os desvios-padrão. Entretanto, essa limitação é decorrente da própria estrutura dos modelos de demanda, que ao estimar por SUR, não permitem que o desenho amostral seja declarado.

\section{Referências}

Allais, O.; Bertail, P.; Nichèle, V. (2010). The effects of a fat tax on French households' purchases: a nutritional approach. The American Journal of Agricultural Economics 92 (1): p. 228-245.

Banks, J.; Blundell, R.; Lewbel, A. (1997). Quadratic Engel curves and consumer demand. The Review of Economics and Statistics, 79 (4): 527-539.

Bertail, P.; Caillavet, F. (2008). Fruit and vegetable consumption patterns: a segmentation approach. The American Journal of Agricultural Economics, 90 (3): 827-842.

Blundell, R.; Robin, J. M. (1999). Estimation in large and disaggregated demand systems: an estimator for conditionally linear systems, Journal of Applied Econometrics (14): 209-232

Brasil. Ministério da Saúde. Guia alimentar para a população brasileira: promovendo a alimentação saudável. Brasília: Secretaria de atenção à saúde, 2005. Disponível em: http://dtr2001.saude.gov.br/editora/produtos/livros/ pdf/05 1109 M.pdf Acesso em: 18 de abril de 2012.

Cash, S. B.; Sunding, D. L.; Zilberman, D. (2005). Fat taxes and thin subsidies: Prices, diet, and health outcomes, Food Economics - Acta Agriculturae Scandinavica, Section C, 2: 167-174.

Claro, R. M. ; Monteiro, C. (2010). A. Renda familiar, preço de alimentos e aquisição domiciliar de frutas e hortaliças no Brasil. Revista de Saúde Pública 44 (6): p. 1014-1020.

Coelho, A. B. A demanda de alimentos no Brasil. 2006. 248 p. Tese (Doutorado em Economia Aplicada). Viçosa: Universidade Federal de Viçosa.

.; Aguiar, D. R. D. De; Eales, J. S. (2010). Food demand in Brazil: an application of Shonkwiler \& Yen Two-Step estimation method. Estudos Econômicos 
40 (1): 186-211.

Cox, T.; Wohlgenant, M (1986). Prices and quality effects in cross-section demand analysis. The American Journal of Agricultural Economics. 68 (4): 908 - 919.

Deaton, A. (1988). Quality, quantity and spatial variation of prices. The American Economic Review 78 (3), p. 418-430, jun., 1988.

.; Muellbauer, J. (1980a). Economics and consumer behavior. New York: Cambridge , 450p.

. An Almost Ideal Demand System. (1980b). The American Economic Review. 70 (3): 312-326.

Drewnowski, A.; Popkin, B. M. (1997). The nutrition transition: new trends in the global diet. Nutrition Reviews 55, (2): 31-43.

.; Darmon, N.; Briend, A. (2004). Replacing fats and sweets with vegetables and fruits: a question of cost. American journal of public health 94 (9): 1555-1559.

Durham, C.; Eales, J. (2010). Demand elasticities for fresh fruit at the retail level. Applied Economics 42 (11): 1345-1354.

Food and Agricultural Organization of the United Nations - FAO. (1997). Implications of economic policy for food security: a training material. Roma: FAO.

Feng, X; Chern, W. S. (2000). Demand for healthy food in the United Sates. Selected paper presented at the meetings of the American Agricultural Economics Association. Disponível em: http://faculty.ksu.edu.sa/62311/Research\%20 Library/23.pdf. Acesso em: 25 de março de 2012.

Hovhannisyan, V.; Gould, B. W. (2011). Quantifying the structure of food demand in China: An econometric approach. Agricultural Economics 42 (1): sup.1-17.

Instituto Brasileiro de Geografia e Estatística - IBGE. (2010a).

(2010a). Pesquisa de Orçamentos Familiares: aquisição domiciliar per capita. Rio de Janeiro: IBGE.

. (2010b) Microdados da POF 2008-2009 (Pesquisa de Orçamentos Familiares). Rio de Janeiro: IBGE.

(2010c). Pesquisa de Orçamentos Familiares: Despesas, rendimentos e condições de vida. Rio de Janeiro: IBGE.

Leifert, R. M.; Lucinda, C. R. de. (2012). Análise dos efeitos de um imposto sobre alimentos engordativos no mercado brasileiro. In: Encontro Nacional de Economia, 40, Porto de Galinhas, 2012. Anais eletrônicos... Porto de Galinhas: ANPEC.

Moschini, G. (1995). Units of measurement and the Stone Index in demand system estimation. American Journal of Agricultural Economics 77: 63-68.

Mutuc, M. E. M.; Pan, S.; Rejesus, R. M. (2007). Household vegetable demand in the Philippines: Is there an urban-rural divide? Agribusiness 23 (4): 511-527.

Organização Mundial da Saúde - OMS. (2002). Reducing risks, promoting healthy life. Paris: WHO.

POI, B. P. (2008). Demand-system estimation: Update. The Stata Journal 8 (4): 554-556. 
Pollak, R. A.; Wales, T. J. (1981). Demographic variables in demand analysis. Econometrica 49 (6): 1533-1551

Rocha, S. (1998). Renda e Pobreza: medidas per capita versus adulto equivalente. Rio de Janeiro: IPEA (Texto para discussão, n. 609).

Sarti, F. M. ; Claro, R. M. ; Bandoni, D. H. (2011) . Contribuições de estudos sobre demanda de alimentos à formulação de políticas públicas de nutrição. Cadernos de Saúde Pública 27 (2): 639-647.

Schmidt, M.i.; Duncan, B.b.; Silva, G.a.; Menezes, A.m.; Monteiro, C.A.; BARRETO, S.M.; CHOR, D.; MENEZES, P.R. (2011). Chronic non-communicable diseases in Brazil: burden and current challenges. Lancet (377): 1949-61.

Shonkwiler, J.; Yen, S. Two-step estimation of a censored system of equations. American Journal of Agricultural Economics, v. 81, n. 4, p. 972-982, Nov. 1999.

Smed, S., Jensen, J. D., Denver, S. (2007) Socio-economic characteristics and the effect of taxation as a health policy instrument. Food Policy 32: 624-639.

Yen, S. T.; Kan, K.; Su, S. (2002). Household demand for fats and oils: two-step estimation of a censored demand system, Applied Economics 34 (14):17991806, 2002.

., Lin, B.; Smallwood, D. M. (2003). Quasi- and simulated-likelihood approaches to censored demand systems: food consumption by food stamp recipients in the United States, American Journal of Agricultural Economics, (85): 458-478.

.; Tan, A. K. G.; Nayga Jr., R. M.(2011). Determinants of fruit and vegetable consumption in Malaysia: an ordinal system approach. The Australian Journal of Agricultural and Resouce Economics (55): 239-256.

\section{Apêndice}




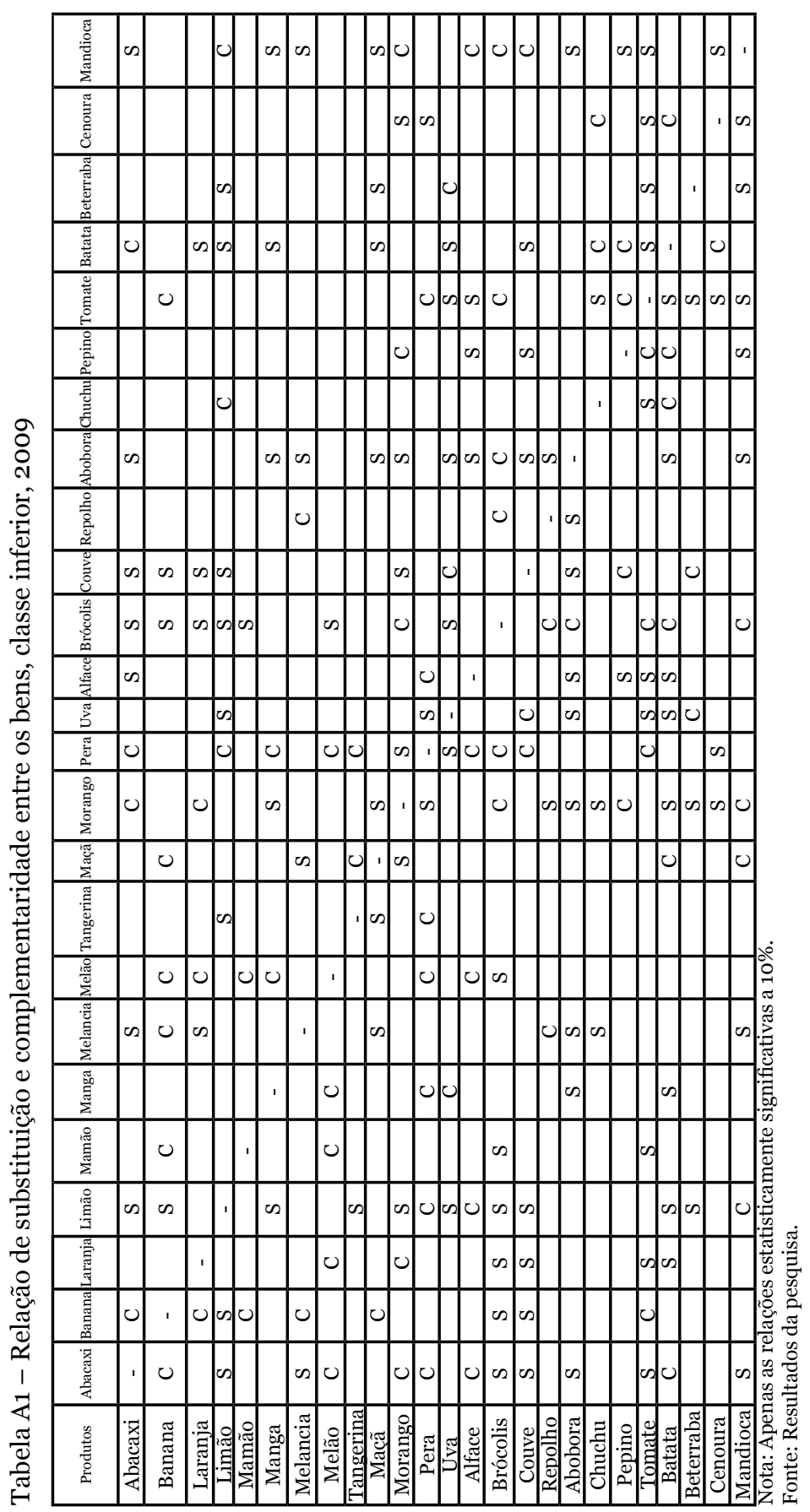




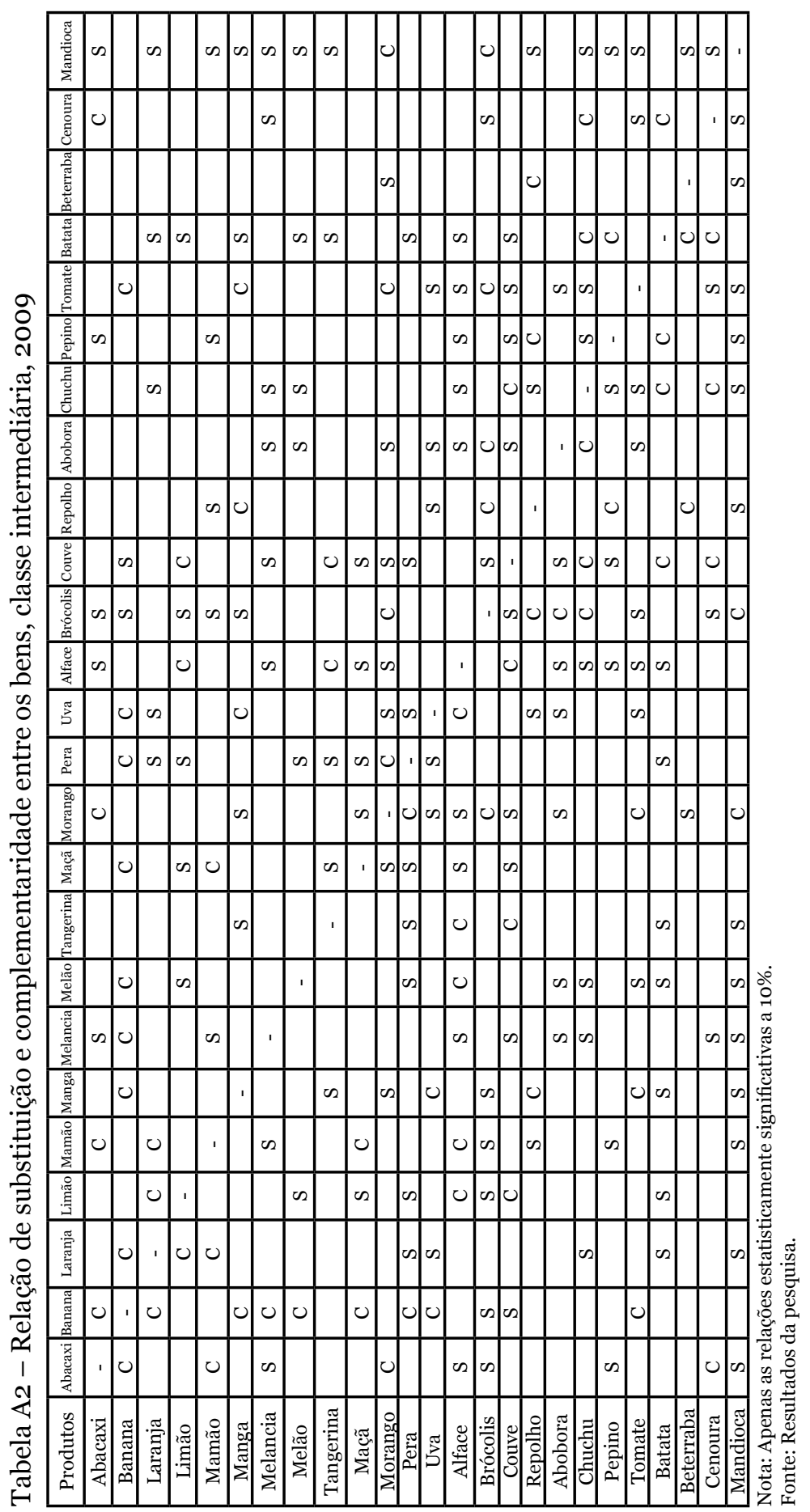




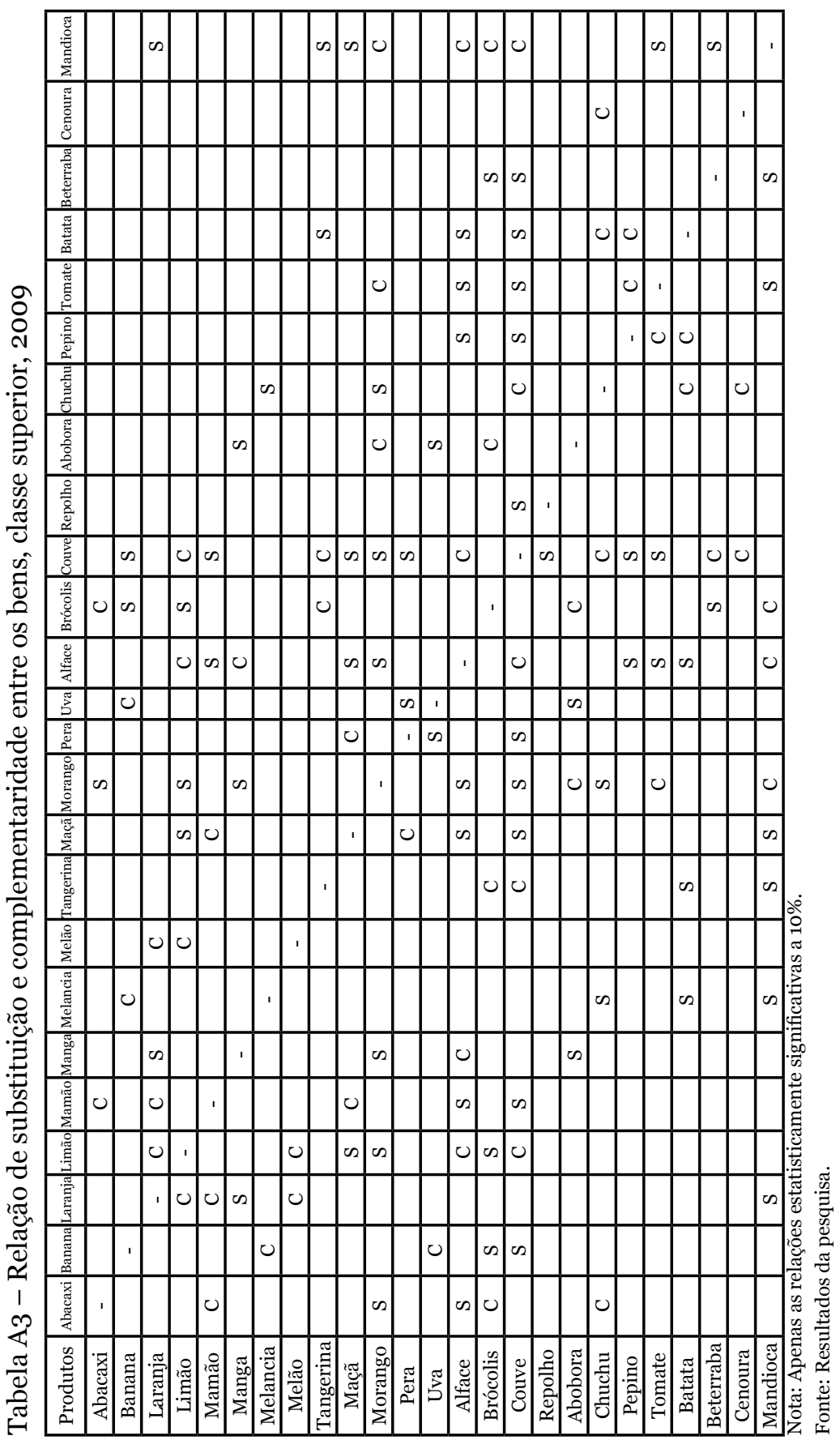




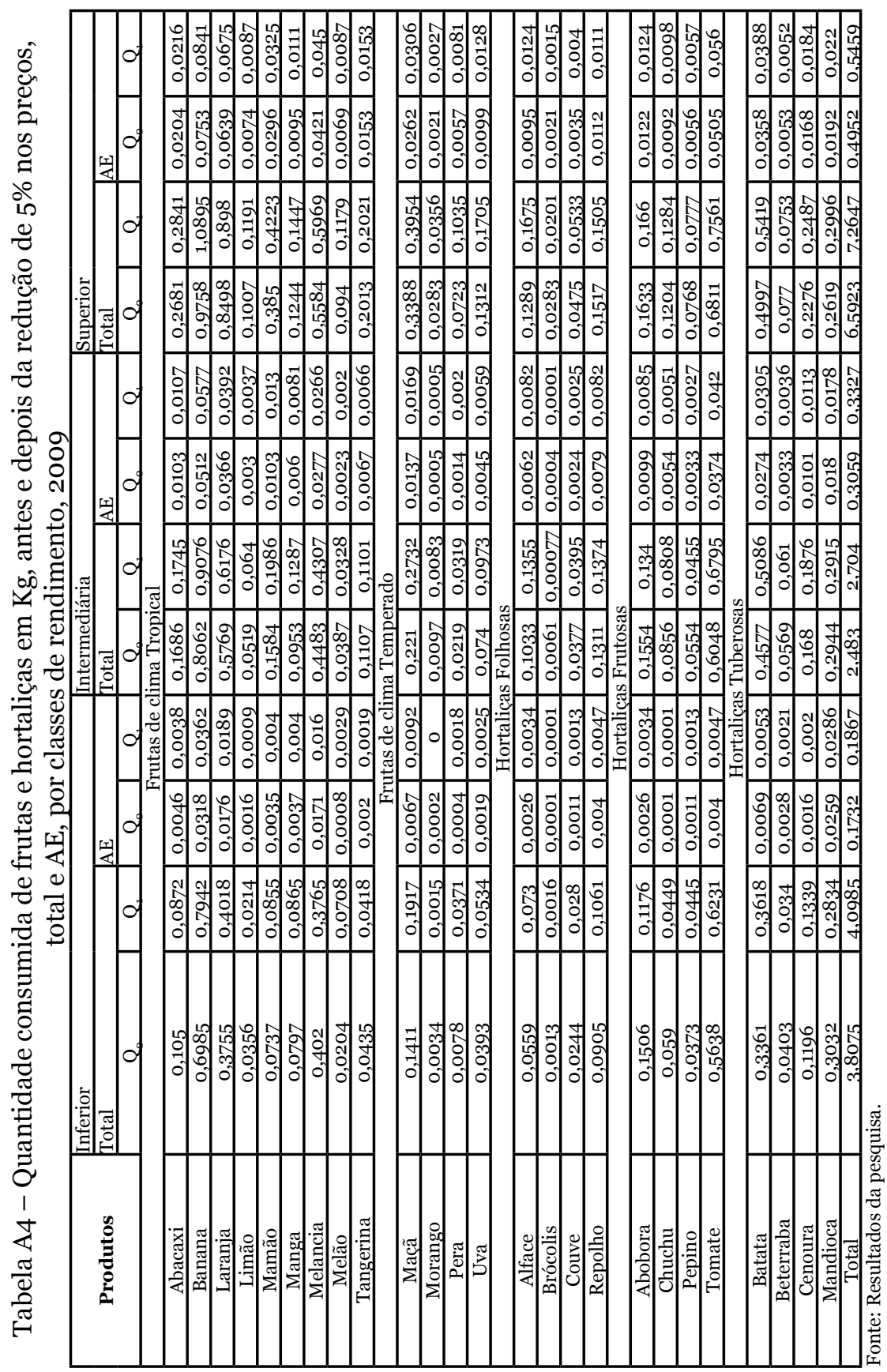


